\title{
Diseño del Sistema de Gestión de Información UNCRISPROCA ${ }^{[19]}$
}

Henry Thyne Taylor ${ }^{[20]}$

yorda Rostran Aragón ${ }^{[21]}$

Gaudi Beer $^{[22]}$

\section{Resumen}

La Cooperativa Unión Cristiana de Productores de Cacao (UNCRISPROCA), se localiza en la Comunidad de Siawas, municipio de La Cruz de Río Grande. Fue fundada en 1997, por 112 personas socias de la comunidad.

La Cooperativa Unión Cristiana de productores de Cacao (UNCRISPROCA), trabaja en la actualidad de manera manual, tiene atraso en la agilización de la indagación de información concerniente a la cooperativa, ellos buscan en archivos impresos en papel y manuscritos toda la información que necesitan para elaborar informes o reportes de la cooperativa, así como averiguaciones de los afiliados, producción, afectaciones, totalidad del acopio de cacao, entre otros.

Con la creación del sistema de gestión de datos para la Cooperativa Unión Cristiana de Productores de Cacao (UNCRISPROCA), se lograrán alternativas tecnológicas para agilizar los procesos de control y registro, minimizando el tiempo de búsqueda y respuesta de información relevante de la cooperativa, con lo cual el trabajo del administrador y el coordinador será rápido y eficaz, esto coadyuvará a una estratégica en la toma de decisión, en cuanto a las afectaciones de la producción.

El sistema diseñado permitirá un registro de los productores que exiten en la zona, las afectaciones a la siembras, ubicaciones de las parcelas, el total de hectáreas de cacao de la cooperativa UNCRISPROCA, permitiendo de esta manera una gestión de información pertinente, eficaz y eficiente, mejorando el proceso en la toma de decisiones.

[19] Monografía realizada con el financiamiento del proyecto PATHWAY II "Support to Graduation Research Projects of Indigenous and Afro Desendant Students in URACCAN, de la Fundación FORD.

[20] Licenciado en Informática Administrativa.

[21] Licenciada en Informática Administrativa.

[22] Ingeniera Agrónoma, tutora de la investigación. 
Palabras claves: Gestión de información, Cooperativa Unión Cristiana de productores de Cacao, Cruz de Río Grande.

\section{Introducción}

La cooperativa Unión Cristiana de Productores de Cacao (UNCRISPROCA), localizada en la Comunidad de Siawas, municipio de La Cruz de río Grande. Está a una distancia aproximada de 40 kilómetros de la cabecera municipal, sus vías de acceso son la acuática y terrestre. Sus coordenadas geográficas corresponden a una latitud: $13^{\circ} 10^{\prime}$ 26.1" y una longitud: $84^{\circ} 08^{\prime} 15.9^{\prime \prime}$.

La comunidad fue fundada en 1960 por unas 10 familias provenientes de San José de los Remates, La Trinidad, Nandaime, Esquipulas y Siuna. Cuando llegaron al lugar, este era conocido con el nombre de Siawas, legado de la lengua de sus pobladores indígenas originarios. Siawas está habitado principalmente por miembros de la etnia mestiza, la principal actividad económica es la agricultura de subsistencia y la ganadería extensiva.

En 1997 finaliza el apoyo de la iglesia Católica, en ese mismo año las productoras y productores observaron un cambio en las plantaciones de cacao, valoraron las técnicas para controlar la monilia, decidieron organizarse para continuar gestionando apoyo en pro del mejoramiento del cacao y con esta idea se formó la Unión Cristiana de Productores de Cacao (UNCRISPROCA), R. L. con 112 socios y socias, agosto del año 1997. Actualmente son 118 personas miembros, de los cuales 27 son mujeres.

En 1998 la Organización Austríaca de Cooperación Para el Desarrollo HORIZONT3000, inició su colaboración con FADCANIC en la Región Autónoma del Atlántico Sur, impulsando un proyecto de emergencia por la afectación que sufrió la población que habita en la cuenca media del río Grande de Matagalpa, por el paso del Huracán Mitch en octubre de 1998 y con el objeto de continuar la asistencia, fortaleciendo los esfuerzos que realiza la cooperativa Unión Cristiana de Productores de Cacao (UNCRISPROCA). R. L. (Montalbán; 2004).

La cooperativa cuenta con una Junta Directiva, una Junta de Vigilancia y un Comité de Medio Ambiente, la cual está conformada con las siguientes personas.

- La Junta Directiva está estructurada de la siguiente manera: Omar Tercero, presidente; Esterlina Rocha, vicepresidenta; Francisco Álvarez, secretario; Arsenio Hurtado, tesorero y Samuel Urbina, vocal. 
- La Junta de Vigilancia está conformada por 3 personas: Catalino Obando Hurtado, coordinador; José Heberto, secretario y Alejandro Hernández, vocal.

- El Comité de Medio Ambiente está conformado por: Agapito Centeno, coordinador; Vicente Cruz, secretario y Feliciano Bello, vocal.

- La Junta Directiva celebra cada final de año su asamblea ordinaria.

La Cooperativa UNCRISPROCA intercambia productos con otras cooperativas de Waslala y río San Juan. Su producción es exportada cuatro veces al año hacia Austria. Tiene 10 años de existencia en la comunidad de la Cruz de río Grande y cuenta con 6 acopios más, cada acopio cuenta son sus herramientas de trabajo (pesas, sacos, probador de humedad, cuchilla para cortar cacao, etc.). Los socios siembran sus propias parcelas y FADCANIC les brinda asesoría, capacitaciones a cada parcela con el apoyo financiero de Horizont3000.

Desde que fue fundada esta cooperativa no ha tenido ningún tipo de estudio sobre la falta de mecanismos efectivos computarizados, que permitan controlar o analizar la sistematización de la producción del cacao.

Con la creación e implementación de este sistema de gestión, se diseñará un registro preciso de todos los productores afiliados y no afiliados a la corporativa, se conocerá la cantidad de manzanas o hectáreas que tienen disponibles y cultivadas, los diferentes tipos de afectación, recolección total de la producción, entre otras informaciones.

En la actualidad los elementos de la vida cotidiana se caracterizan por el ritmo del desarrollo tecnológico. "Con ella se marcha hacia una nueva sociedad donde el eje de la productividad no será la cantidad de personas que producen, sino el conocimiento tecnológico y la creatividad de la sociedad".

\section{Objetivos}

\section{Objetivo General:}

Creación de un sistema de gestión informativo en la producción de la cooperativa Unión Cristiana de Productores de Cacao -UNISCRISPROCA-, en la comunidad de Siawas, Municipio de la Cruz de río Grande, RAAS, Nicaragua, 2008. 


\section{Objetivo Específicos:}

1. Identificar las variables y relaciones que conformaran el sistema.

2. Definir la plataforma que se utilizará para el sistema.

3. Elaborar un sistema de gestión de información.

\section{Revisión de Literatura}

Los sistemas de información que logran la automatización de procesos operativos dentro de una organización, son llamados frecuentemente sistemas transaccionales, su función primordial consiste en procesar pagos, cobros, pólizas, entradas, salidas, etc. Por otra parte, los sistemas de información que apoyan el proceso de toma de decisiones son los sistemas de soporte a la toma de decisiones, sistemas para la toma de decisión de grupo, sistemas expertos de soporte a la toma de decisiones y sistema de información para ejecutivos. El tercer tipo de sistema, de acuerdo con su uso u objetivos que cumplen, es el de los sistemas estratégicos, los cuales se desarrollan en las organizaciones con el fin de lograr ventajas competitivas, a través del uso de la tecnología de información

Algunos sistemas de gestión de acopio son: SEGES: Un sistema de gestión diseñado y pensado especialmente para la administración de acopio, adaptado a las necesidades de comunicación de hoy y a la dinámica del trabajo que requiere el mercadeo.

Physis acopio y consignación: Es la solución integral para empresas que se dedican a los negocios de compra/venta de agroquímicos, acopio de cereales y consignación de hacienda. Prevé la consolidación completa de todos los circuitos, integrando el manejo de fondos, las cuentas corrientes, la contabilidad y los impuestos nacionales, provinciales y los aplicativos de ONCCA y por último cierra el circuito con la comercialización con el corredor, exportador, etc.

(http://www.physis.com.ar/new.asp 04 de junio 2008,1:50P.m)

\section{Materiales y Método}

Considerando los principios científicos y humanistas de la URACCAN, en la implementación de la metodología se integran todas las variables: interculturales, género, generacional y la sostenibilidad. 
1. Análisis del sistema: Se realizaron los análisis de las variables ocupadas en el desarrollo, para determinar la estructura de tablas y los tipos de variables implementadas en el sistema. Se realizaron entrevistas directas con el coordinador, esta herramienta logró identificar de forma directa la necesidad del acopio.

2. Diseño del sistema: Consistió en la elaboración física de la base de datos tomando como referencia las variables analizadas. Se identificaron los datos necesarios, se definió la composición y estructura de cada interfaz. Esto determinó la forma de la base de datos, el lenguaje usado y finalmente la estructura de los informes procesados de los datos ingresados.

3. Salidas del sistema: Los informes finales representados en el sistema, es el procesamientos de los registros integrados. Éstos se presentan de forma impresa o digital, permitiendo al usuario disponer de la información en cualquier momento.

\section{Resultados y Discusión:}

\section{Variables que conforman el Sistema}

Como resultado del análisis del formulario usado por el ingeniero Guillen Luna para llevar un control de producción, se logró identificar las siguientes variables:

Tabla: Registro del productor.

- Nombre (tipo: carácter).

- Apellido (tipo: carácter).

- Edad (tipo numérico, longmax 2).

- Sexo (tipo: carácter, combobox con 2 ítems).

- Masculino.

- Femenino.

- Comunidad (tipo:carácter).

- Acopio (tipo carácter, combobox con 6 ítems).

- Etnia (tipo carácter, combobox con 4 ítems).

- Creole.

- Mestizo. 
- Miskitu.

- Sumu.

- Afiliado ((tipo: carácter, combobox con 2 ítems).

- $\mathrm{Si}$

- No

- Produce cacao (tipo: carácter, combobox con 2 itmes).

- Si

- No

Tabla: siembra

- Hectáreas sembradas (tipo:numérico, longmax 3).

- Cantidad de árbol (tipo: numérico, longmaz 3).

- Estado de la planta (combobox con 3 items).

Tabla: producciones varias

- Producto (tipo carácter).

- Hectárea sembrada (tipo numérico, longmax 3).

Tabla: recolecta

- Ubicación (tipo numéricos, longmax 7).

$-\mathrm{X}$

$-\mathrm{Y}$

- Afectación a la siembra (combobox 2 item).

- $\mathrm{Si}$

- No

Tabla: Afectación

- Plaga (tipo carácter).

- Cantidad de árbol afectado (tipo numérico, longmax 3).

- Estado de afectación (combobox con 4 items).

- Normal.

- Poco afectado. 


\section{SISTEMAS DE INFORMACIÓN SOCIOAMBIENTAL}

- Afectado.

- Altamente.

- Afectado.

Tabla: control de la plantación.

- Nombre (tipo: carácter).

- Apellido (tipo: carácter).

- Acopio (tipo carácter, combobox con 6 ítems).

- Id parcela (tipo: Numérico).

- Tipo de control (Tipo: carácter, Text box).

- Estado de cumplimiento(combo box 2Items).

- Cumplida.

- No cumplida.

- Fecha de cumplida.

- Observación (Tipo: carácter).

Una vez identificadas las variables se establecieron las debidas relaciones de las mismas, como se muestran en las figuras 1 y 2. 


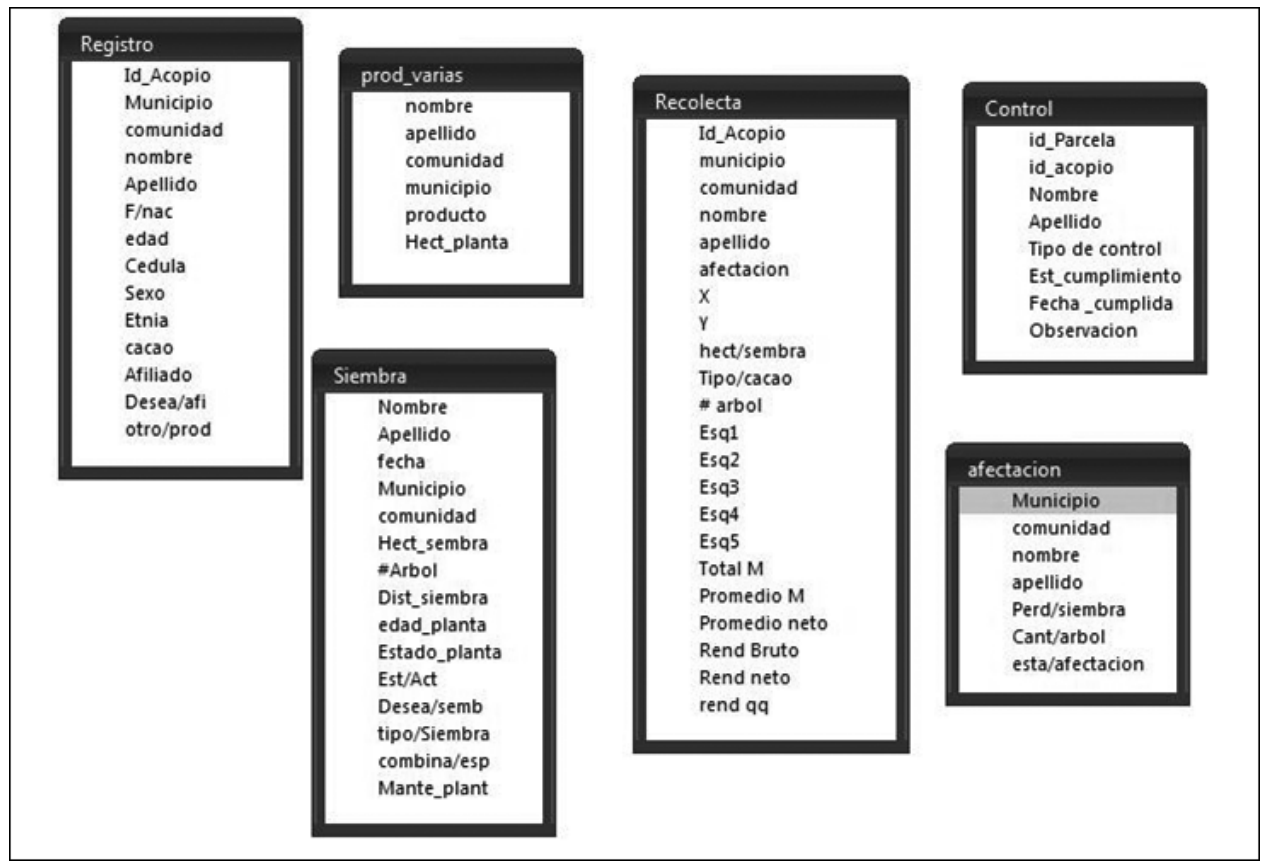

Figura 1. Estructura de la Base, Tablas Microsoft Access 2007

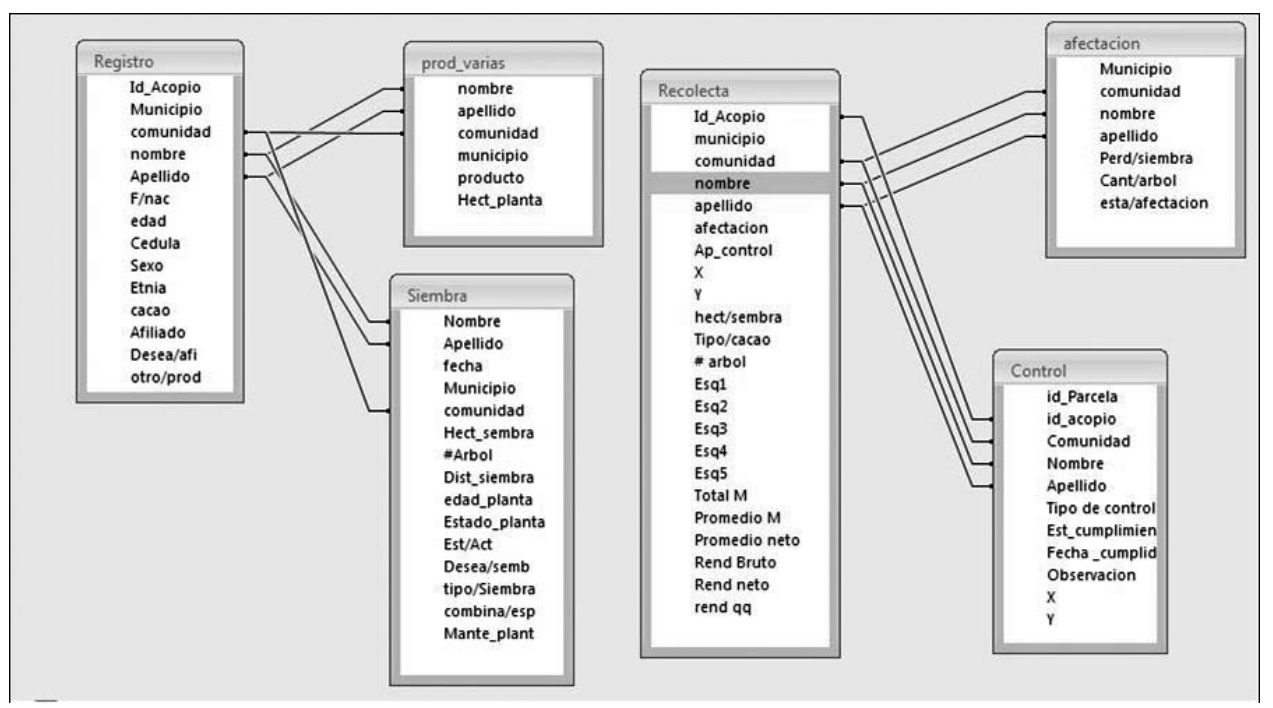

Figura 2. Relaciones entre las tablas. Microsoft Access 2007 


\section{Plataforma a utilizar}

Se optó en ocupar dos plataformas en la construcción del sistema, Microsoft Access 2007 y Microsoft Visual Basic 6.0 La elección de Microsoft Access 2007 se basó en su capacidad de integrarse con el resto de las aplicaciones Microsoft. Asimismo, dado que es un sistema de seguridad de cifrado bastante primitivo, puede ser la respuesta a pequeños y medianos proyectos de programación. Para el caso de Microsoft Visual Basic 6.0 su elección fue la rapidez con la que se puede diseñar un programa que utilice base de datos sencillos, además de la abundancia de programadores en este lenguaje. Por último, dado que su lenguaje de programación es reconocido mundialmente, se pueden encontrar soluciones a los problemas que se presenten en la creación del sistema, de una manera sencilla y segura.

\section{Sistema de Gestión de Información}

El propósito general de los sistemas de gestión de base de datos, es manejar de manera clara, sencilla y ordenada, un conjunto de datos que posteriormente se convertirán en información relevante.

En el diseño del sistema se consideraron los efectos que se pueden presentar en la creación del nuevo sistema en el entorno a funcionar, adecuando los criterios de diseño a las características del mismo. En este contexto, adquiere una importancia creciente la adaptación de todo sistema - producto a las capacidades de las personas que van a utilizarlo, de forma que su operación sea sencilla, cómoda, efectiva y eficiente.

Se tomó en cuenta la ergonomía, que tiene por objeto la optimización del entorno hombre-máquina. En la actualidad la relación hombre - máquina ha pasado a intervenir con fuerza en todos los procesos cognitivos (análisis, interpretación, decisión, comunicación y representación del conocimiento). Así, con respecto al diseño de herramientas software, la ergonomía tiene mucho que decir en relación con la disposición de informaciones en pantalla, profundidad de menús, formato de íconos, nombres de comandos, control de cursores, tiempos de respuesta, manejo de errores, estructuras de datos, utilización de lenguaje natural, etc.

Considerando estos factores, decidimos que el entorno tenía que ser lo más amigable posible, sin tantos procedimiento para tener una respuesta del sistema, ya que implicaría un tiempo de adaptación más largo, para la aceptación de la base de datos, además que el usuario se podía acoplar rápidamente al sistema. 


\section{Interfaz}

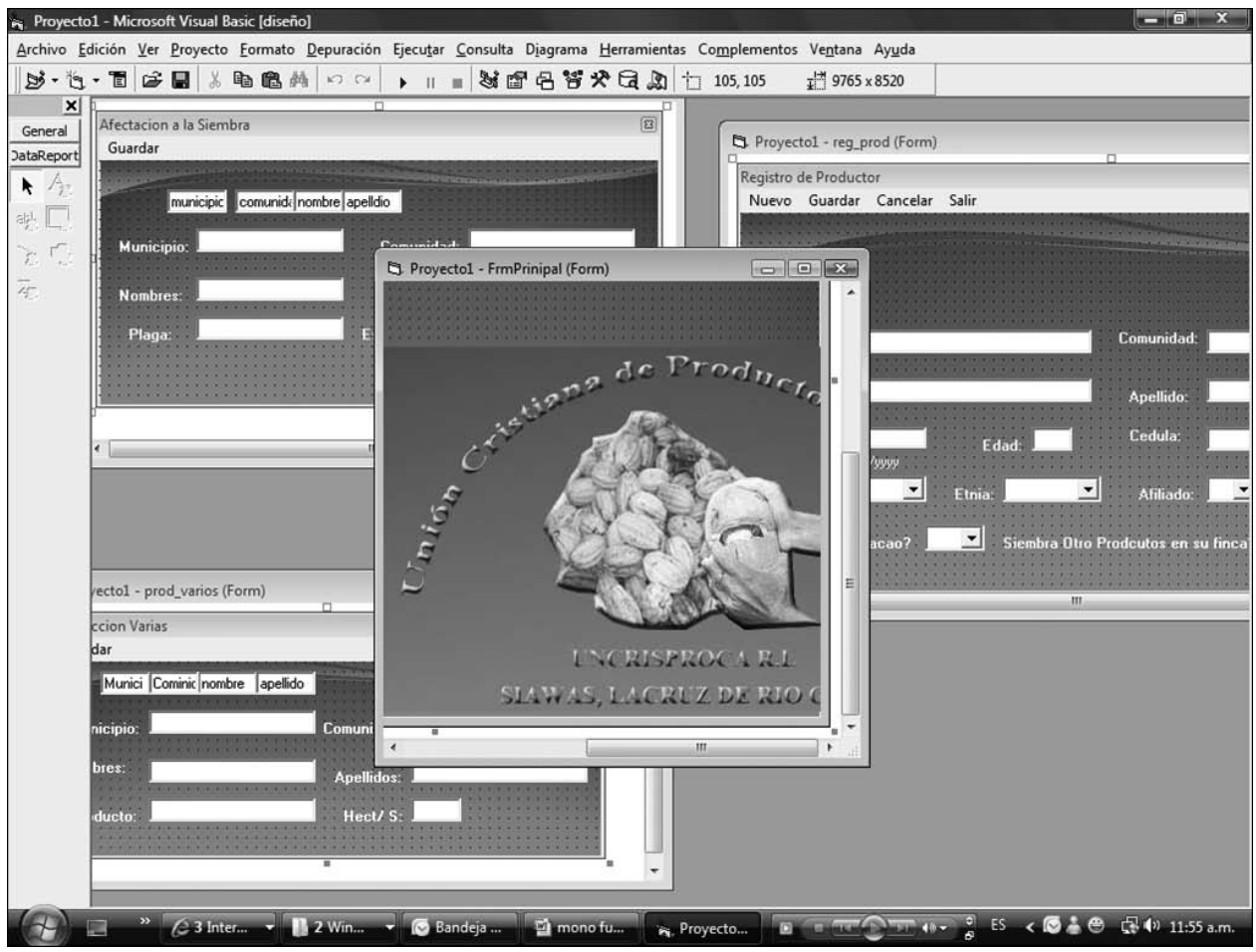

Figura 6. Diseño de Interfaz en modo de diseño. Visual Basic 6

Se diseñó un interfaz amigable, se buscó un color atractivo que permitiera al usuario poder trabajar por las noches, ya que hay colores que no le permiten al usuario trabajar en la oscuridad y perjudican los ojos. El entorno lleva botones con nombres que se ven y se podrá interactuar con ellos tales como: imprimir, nuevo, salir, cancelar, mensaje de criterios, entre otros. 


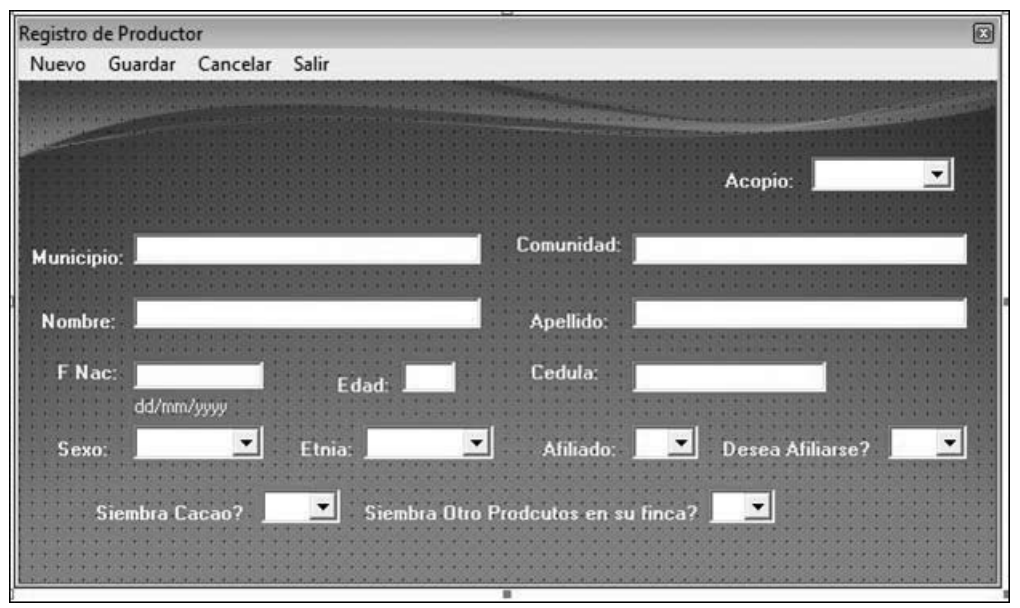

Figura 7. Interfaz Amigable. Visual Basic 6.

\section{Elaboración del Sistema}

El sistema tiene varios formularios amigables y sencillos de llenar, ya que dispone de campos que contienen opciones de respuestas, la selección de una de las alternativas nos brinda una respuesta del sistema, que puede trasladarnos a otro formulario para completar. También por la selección de un botón, nos puede dar una opción que puede ser guardar, eliminar o salir completamente del sistema.

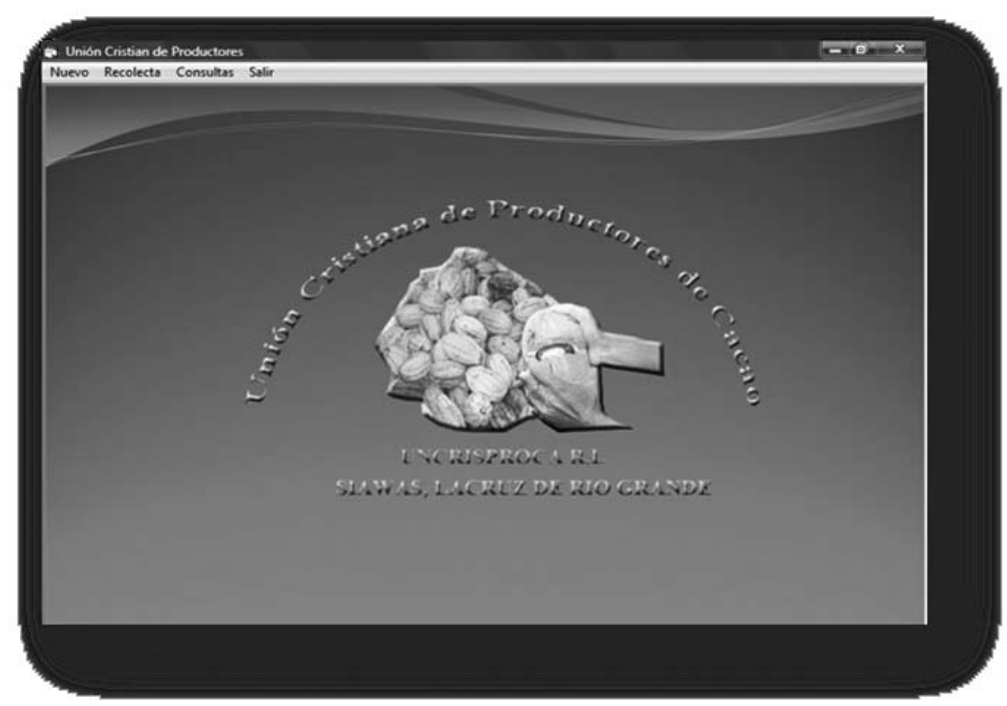

Figura 8. Formulario Principal. Sistema UNISCRIPROCA. 


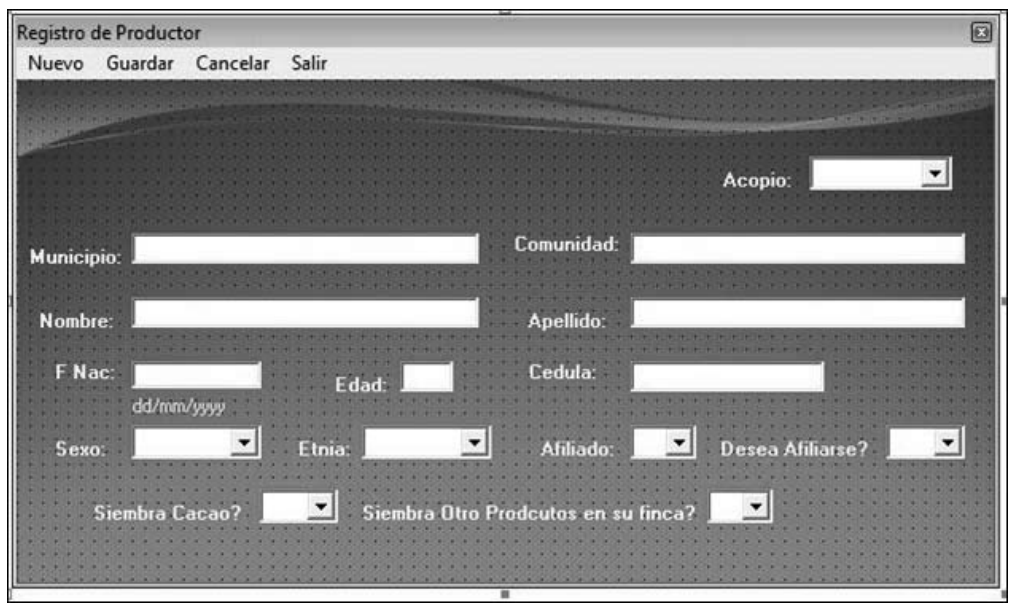

Figura 9. Formulario de registro de productor. Sistema Unión Cristiana de Productores (UNCRISPROCA)

\section{Consultas}

En caso de los datos procesados, el sistema cuenta con un formulario de consultas en el cual el usuario puede consultar datos predefinidos: total de hectáreas, cantidad de productores por acopios, ubicación de las parcelas de forma general o detallada, cuenta con consultas digitales e impresas. Si el usuario así lo desea, cuenta con exportación de ubicaciones de las parcelas de cacao, obteniendo el resultado en el programa de "Microsoft Office Excel", independientemente de la versión que tiene el usuario instalada en su equipo. 


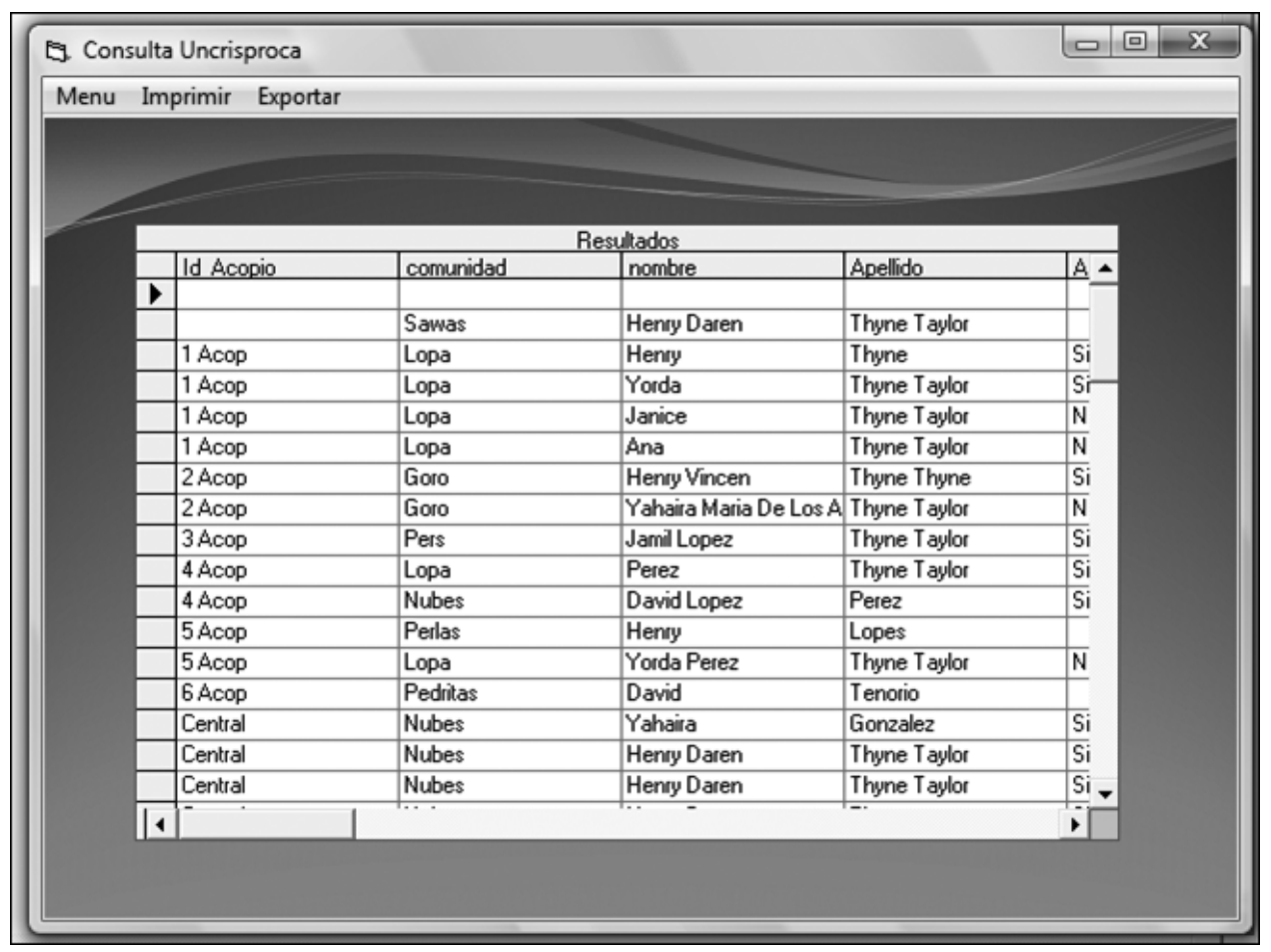

Figura 10. Consulta General de productores por comunidad y acopio Forma Digital. Sistema Unión Cristiana de Productores (UNCRISPROCA). 


\begin{tabular}{|c|c|c|c|c|}
\hline \multirow[b]{2}{*}{ Id_Acopio: } & \multicolumn{3}{|c|}{ Uaion Cristian de Productore de Cacac } & \multirow{3}{*}{ Afliado: } \\
\hline & comunidad: & nombre: & Apellido: & \\
\hline & Sawas & Henry Daren & Thyne Taylor & \\
\hline $1 \mathrm{Acop}$ & Lopa & Henry & Thyne & $\mathrm{Si}$ \\
\hline $1 \mathrm{Acop}$ & Lopa & Yorda & Thyne Taylor & $\mathrm{Si}$ \\
\hline $1 \mathrm{Acop}$ & Lopa & Janice & Thyne Taylor & No \\
\hline $1 \mathrm{Acop}$ & Lopa & Ana & Thyne Taylor & No \\
\hline $2 \mathrm{AcOp}$ & Goro & Henry Vincen & Thyne Thyne & Si \\
\hline $2 A C O P$ & Goro & Yahaira Maria De Los & Thyne Taylor & No \\
\hline 3 AcOP & Pers & Jamil Lopez & Thyne Taylor & Si \\
\hline $4 \mathrm{Acop}$ & Lopa & Perez & Thyne Taylor & Si \\
\hline $4 \mathrm{ACOP}$ & Nubes & David Lopez & Perez & $\mathrm{Si}$ \\
\hline 5 AcOp & Perlas & Henry & Lopes & \\
\hline 5 Acop & Lopa & Yorda Perez & Thyne Taylor & No \\
\hline 6 Acop & Pedritas & David & Tenorio & \\
\hline Central & Nubes & Yahaira & Gonzalez & $\mathrm{Si}$ \\
\hline
\end{tabular}

Figura 11. Informe preliminar Consulta de productores registrados por comunidad. Sistema Unión Cristiana de Productores de Cacao (UNCRISPROCA).

\section{Exportación de Datos}

Con la exportación de los datos a Microsoft Excel, se pretende contar con la ubicación de las parcelas de los productores de cacao, ya que con dichas coordenadas se puede geo-referenciar, ocupando un software de cartografía tal como ArcGis o ArcView , que son los más ocupados y mencionados aquí en la región autónoma y estas utilizan enlaces con EXCEL. 


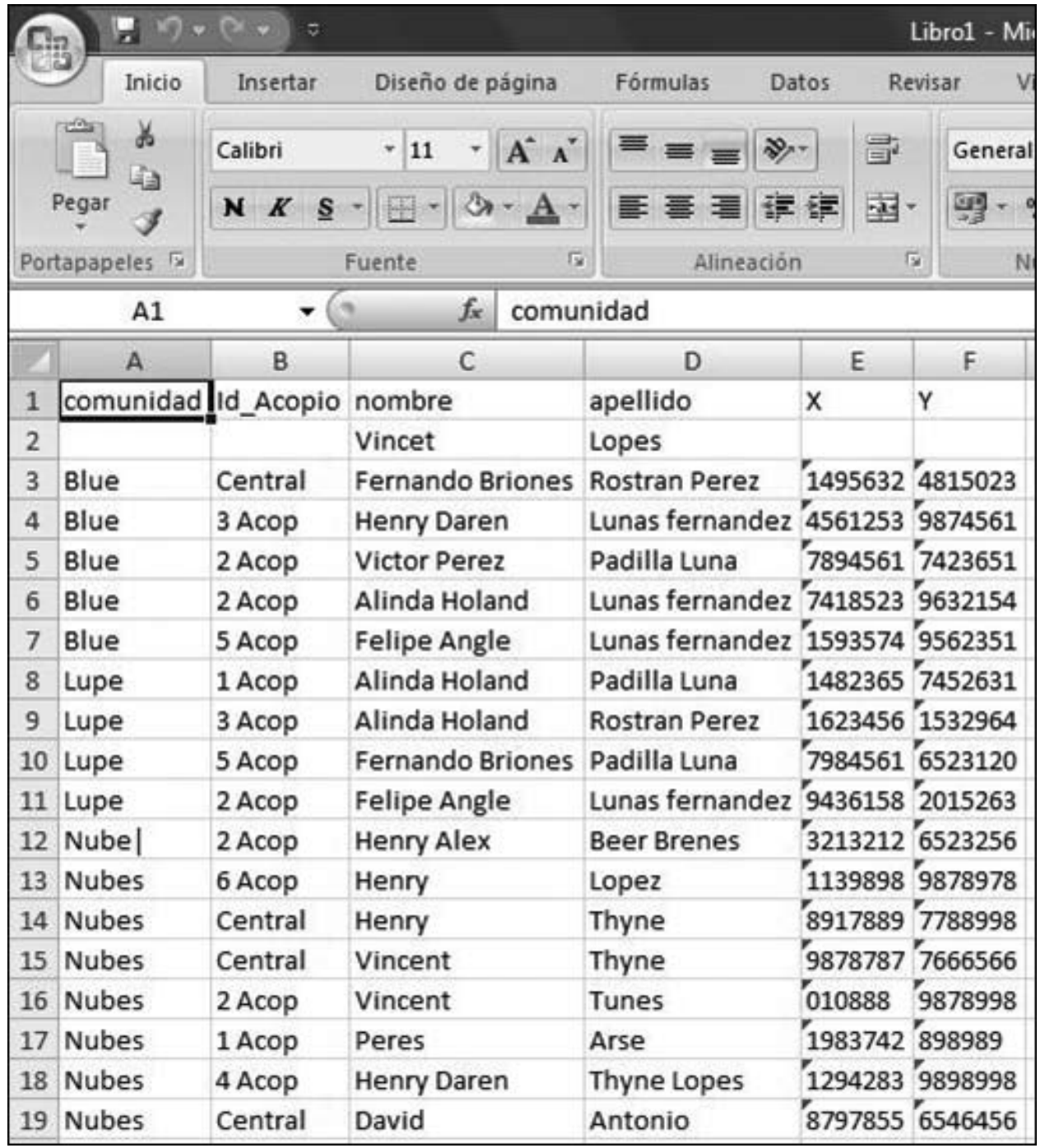

Figura 12. Consulta de ubicación de las fincas datos exportados. Sistema Unión Cristiana de Productores de Cacao (UNCRISPROCA).

\section{Conclusiones:}

1. La Cooperativa Unión Cristiana de Productores de Cacao (UNCRISPROCA), trabaja en la actualidad de manera manual, tiene un gran atraso en la agilización de la 
indagación de información concerniente a la cooperativa, ellos buscan en archivos impresos en papel y manuscritos toda la información que necesitan para elaborar informes o reportes de la cooperativa, así como averiguaciones de los afiliados, producción, afectaciones, totalidad del acopio de cacao, entre otros.

2. Con la creación del sistema de gestión de datos para la Cooperativa Unión Cristiana de Productores de Cacao (UNCRISPROCA), se efectivizará la búsqueda de alternativas tecnológicas para agilizar los procesos de control y registro, minimizando el tiempo de búsqueda y respuesta de información relevante de la cooperativa, logrando que el trabajo del administrador y el coordinador sea más rápido y eficaz, lo cual coadyuvará a una estratégica en la toma de decisión, en cuanto a las afectaciones de la producción.

3. También podrán valorar qué otro producto que está en cultivo puede ser ofertado en el mercado nacional e internacional, ampliando de esta manera la diversificación de los productos de la cooperativa, mejorando la calidad de vida de los productores y fortaleciendo la cooperativa.

4. Tanto el administrador, coordinador y los responsables de cada acopio pueden alimentar su sistema, con información nueva y unificar la información de cada acopio en un solo archivo, al finalizar cada meses.

\section{Recomendaciones:}

1. Se recomienda la implementación del sistema de gestión en la Cooperativa Unión Cristiana de Productores de Cacao (UNCRISPROCA), debido a que el programa facilita la gestión de información que se necesita para la toma de decisión y búsqueda de nuevos productos con los cuales se pueda potenciar el mercado nacional e internacional.

2. La base de datos se pude instalar en varias PC o portátiles, para que el sistema pueda ser utilizado en los diferentes acopios de la cooperativa, en los puntos estratégicos del municipio.

3. Este sistema no necesita de grandes conocimiento de computación, ningún especialista que venga a instalar dicho sistema, ya que cuenta con interfaz amigable en donde el usuario se puede guiar con mucha facilidad.

4. La base de datos se puede actualizar con la función de copiar y pegar, que es el paso más común utilizado actualmente en computación.

5. Considerando la importancia a nivel internacional de los Sistemas de Información Geográficas, se le recomienda al ingeniero Guillen Luna, adaptar 
el concepto conocido como agricultura de precisión, el cual consiste en vincular la tecnología SIG a los sistemas de producción, reportando beneficios no sólo económicos sino socio ambientales.

Se recomienda instalar el sistema en una computadora con las siguientes características, $40 \mathrm{~GB}$ en disco duro, $256 \mathrm{Mb}$ en RAM como mínimo y con un procesador de $1.8 \mathrm{GHZ}$ Intel.

\section{Lista de referencias}

Proyecto Corredor Biológico del Atlántico, Componente de Planificación y Monitoreo. (2003). Plan De Desarrollo Comunitario de Siawas, Municipio De La Cruz De río Grande, Región Autónoma del Atlántico Sur, Nicaragua.

Sitios Web http://es.wikipedia.org/wiki/Sistema (27 junio 2008, 4:57 p.m.)

http://es.wikipedia.org/wiki/ Sistema Información Geográfica (27 junio 2008, 5:00 P.M)

http://es.wikipedia.org/wiki/DBMS (27 junio 2008, 4:57 P.M )

http://www.monografias.com/trabajos7/sisinf/sisinf.shtml\#tusi , 28 junio 2008 ,6:15PM

http://ipmworld.umn.edu/cancelado/Spchapters/Hutchins3Sp.htm 04 de junio 2008, 2:15 P.M

http://www.seges.com.ar/ 04 de junio 2008, 1:40 P.m

http://www.physis.com.ar/new.asp 04 de junio 2008,1:50P.m 